\title{
Failure Causes in Total Hip Replacements: A Review
}

\author{
Sipek KT ${ }^{1}$, Lyvers $\mathrm{ME}^{1}$ and Mathew $\mathrm{MT}^{1,2 *}$ \\ ${ }^{1}$ Department of Biomedical Science, UIC School of \\ Medicine, USA \\ ${ }^{2}$ Department of Orthopedic Surgery, Rush University \\ Medical Center, USA
}

*Corresponding author: Mathew MT, Department of Orthopedic Surgery, Rush University Medical Center, USA and Department of Biomedical Science, UIC School of Medicine, USA

Received: February 09, 2018; Accepted: March 08, 2018; Published: March 15, 2018

\begin{abstract}
There are many different materials, shapes, and sizes of hip implants that surgeons should research to decide which is best for each patient. There have been advances in these prostheses in the previous 30 years that allow for the most positive result. Studies have been completed by physicians and research scientists to assist in educating the medical community regarding new advancements in the subject of hip replacements. This paper will discuss these advances and the factors affecting the success rate of hip implants. Some of these include smoking, obesity, metal allergy, corrosion, and medications. Each failure mode has previous research completed to expand upon the underlying causes and what could be done differently to ensure a more positive outcome. This research also discusses the successful aspects of the implants that should become more commonplace for total hip arthroplasties. A recurring failure was due to metal allergy caused by metal particles entering the body. Patient factors also have an influence on the lifespan of the implants. Older patients generally have a lowered bone density which can lead to the implant becoming loose inside the bone. The implant shape and orientation can affect the prosthesis success as well. Studies found that larger head size and a more vertical component angle led to a greater failure rate. This could be due to greater frequency of dislocations due to improper size and orientation for the specific patient.
\end{abstract}

Keywords: Failure processes; Corrosion; Tribocorrosion; Types of THR

\section{Introduction}

Total hip arthroplasties have been an effective treatment choice for arthritis and other disorders for over a century. The first attempt at a hip replacement occurred in Germany in 1891. The modern, low friction arthroplasty, on which current devices are based, was invented by Sir John Charnley in the early 1960's [1]. As more knowledge and experience was gained, there have been modifications to improve the functionality and lifespan of hip implants. These have included material, shape, angle, and size changes. Especially in the last 30 years, many physicians have been researching and completing studies on the cause of hip implant failures so that improvements can be applied, and the different types of implants can be compared. There are also outside factors that affect the success and lifespan of the hip implant, which includes patient age, gender, and surgical technique. Each patient has individual risk factors that can influence the success of the implant. Some of these involve medication use, smoking, obesity, and bone density. This information will give insight to the medical and engineering communities on what aspects of the hip implant can be improved.

\section{Main Causes of Hip Implant Faillure}

Some of the most prevalent reasons that hip implants fail are due to patient smoking and medication use that can cause infection at the implant site (Figure 1). With hip prostheses containing metal components, patient hypersensitivity can be a factor in the success of the implant. This can lead to pseudotumors in the tissues surrounding the implant and subsequent failure. As the patient becomes active following surgery, there is the possibility of corrosion between the different interfaces in the implant.

\section{Corrosion}

Corrosion occurs when fluids encounter the materials in the hip implant. This can include the synovial fluid and is most common in metals. The deterioration of the material is due to chemical and electrochemical reactions. Pitting, intergranular and galvanic corrosion are the most common forms of corrosion observed in hip implants.

\section{Tribocorrosion at interfaces}

Tribocorrosion is the combination of wear and corrosion transforming the material interfaces. It is caused by both the chemical and mechanical interactions on the materials. There are three different interfaces in the hip implants: head-cup (sliding-corrosion), head-neck (modular junction: fretting-corrosion), and stem-bone (modular junction: fretting-corrosion).

\section{Medication}

Certain medications that the patients are taking can affect the success rate of the hip implant by leading to an increased risk of infection. Some medications, such as diuretics and bisphosphonates, also alter the bone density which can cause more fractures near the implant.

\section{Smoking}

Patient smoking decreases the lifespan of the hip implant because of the slowed rate of wound healing. This can lead to a post-operative infection near the implant which can then cause implant failure. It can also affect how well the bone bonds to the stem of the implant.

\section{Hypersensitivity}

As the patient resumes activity after the total hip arthroplasty, 


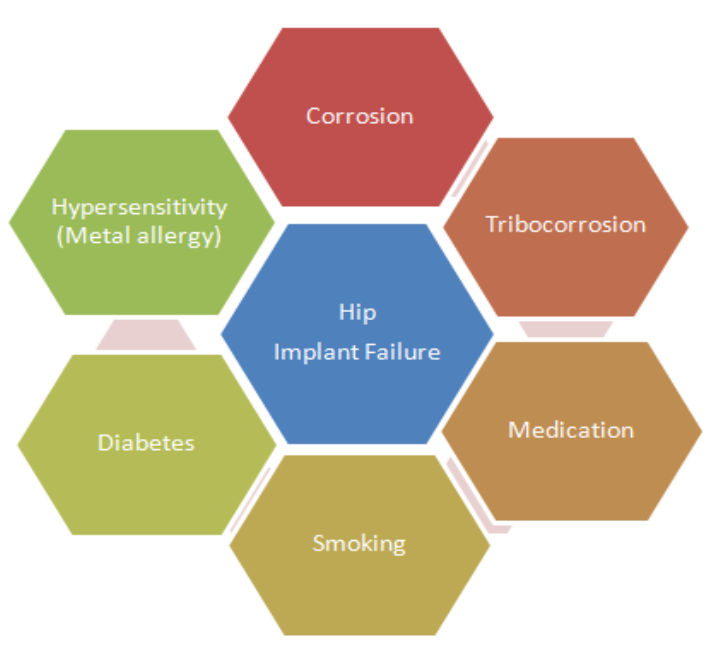

Figure 1: Possible Causes of Implant Failure.

metal particles may be released and enter the surrounding tissues. This may cause a pseudotumor to occur near the implant due to an increased metal ion concentration in the body. Also, some patients may be sensitive to certain metals and can be affected by the implant material.

\section{Implant particle release}

A more uncommon but serious failure method of hip implants involves metal particles loosening from the implant and entering the nearby tissue or bloodstream. In 1985, this issue was researched indepth. Four hip prostheses and surrounding tissues were examined, and it was determined that each had an unusually high concentration of metal due to corrosion of the hip implant's femoral stem [2]. These implants were composed of a metal stem and plastic cup. The removed prostheses showed wear on the femoral stem consisting of scratches on the metal. The cells taken from the body in all four cases displayed evidence of cell necrosis, and the macrophages were in poor condition. This led to macrophage-related bone resorption, which caused loosening of the joint implant and, ultimately, failure of the prosthesis.

A literature search completed between 2011 and 2013 reviewed the effects that cobalt containing hip implants had on the patients [3]. One of the issues with cobalt hip prostheses was determined to be pseudotumors forming near the implant. These are cystic masses that form but do not cause infections or cancer. Another effect found was developing sensitivity to cobalt or chromium. Some studies determined that these two adverse reactions do not correlate to each other; the pseudotumors do not increase the likelihood of cobalt sensitivity. This study found that monitoring cobalt levels in patients with metal hip implants are helpful in screening for negative systemic responses to cobalt.

\section{Failure of different types of hip implants}

The four main types of hip implants include metal-on-metal, metal-on-polyethylene, metal-on-ceramic, and ceramic-on-ceramic (Figure 2). Currently, metal-on-ceramic and metal-on-polyethylene are the most popular types because there is a greatly reduced risk of metal particle release into the surrounding tissues. Hip implants include three different components: the cup, the head, and the stem.
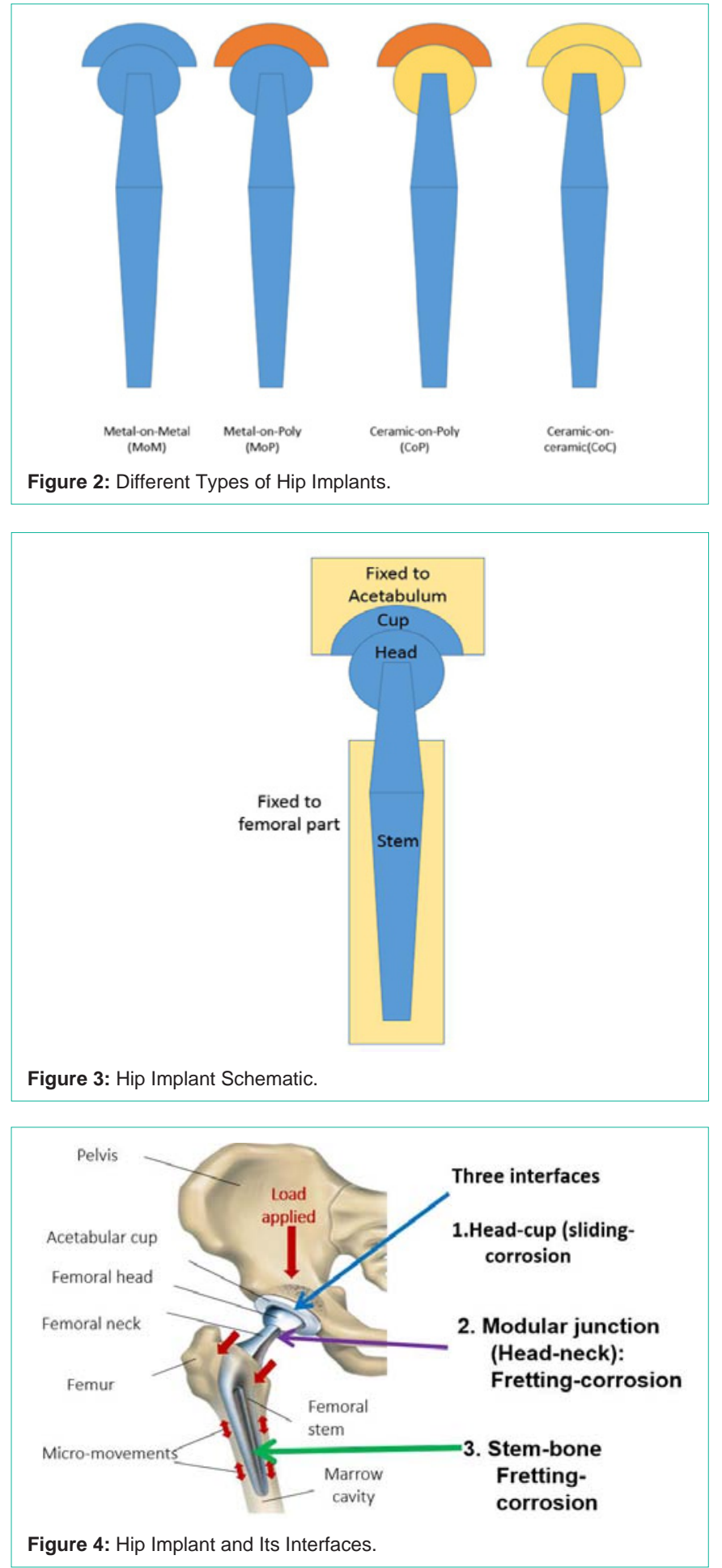

The cup of the prosthesis is fixed to the acetabulum, while the stem is inserted into the femur (Figure 3). The three components make up the three interfaces of the hip implant. The head-cup interface is also referred to as the modular junction and is the point at which most wear occurs. The modular junction and the stem-bone interface undergo fretting corrosion, which is due to micromotions in the implant. The head-cup interface experiences are sliding corrosion from the rotation of the hip (Figure 4). 


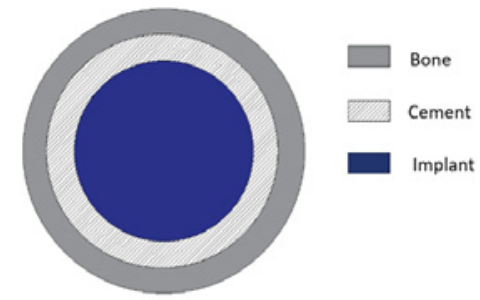

(a)

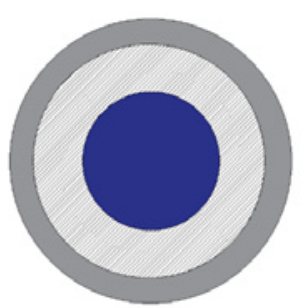

(b)
Figure 5: Model of the (a) 15mm prosthesis and (b) $9 \mathrm{~mm}$ prosthesis [6]

\section{Ceramic hip implant failure}

The purpose of one study done in 1998 was to determine the incidence of osteolysis in patients who had a ceramic hip implant. Of the 103 hip prostheses in 93 patients, only 43 implants were determined stable upon follow up [4]. In this study, 22 percent of the patients showed evidence of osteolysis not only in the femur, but also in the pelvis. The removed ceramic femoral heads and parts of the acetabulum showed indication of damage. In the areas of wear on the ceramic implant, macrophages were found, which indicated a possible site of infection. This could have led to the failure of the hip implant.

In 2013, research was done to analyze the failure of ceramicon-ceramic hip replacements. There were 35 implants retrieved from patients, and each femoral head component showed evidence of wear and damage [5]. The patients that required revision surgery did so due to squeaking with pain, fracture of the acetabular liner, or infection. Squeaking may have been caused by the placement angle or size of the implant along with the demographics of the patient including age, weight, and activity level. In the cases of the fractured acetabular liner, negative effects could have been due to ceramic particles entering the surrounding tissues. Another issue with ceramic-on-ceramic replacements was impingement of the acetabular cup caused by improper placement of the acetabular liner. Both studies demonstrated failures that occurred with ceramic-onceramic hip implants, but they suggest that it is still a preferred choice for younger and more active patients.

\section{Cemented hip implant failure}

A 1994 study focused on strengthening the tip of the prosthesis to prevent loosening by increasing the cement thickness. The implant was modeled with a cement thickness of $2 \mathrm{~mm}$ and $5 \mathrm{~mm}$ by decreasing the prosthesis diameter from $15 \mathrm{~mm}$ to $9 \mathrm{~mm}$ [6] (Figure 5).The models were loaded with both tensile and shear stresses to mimic the loads applied during gait. By increasing the cement mantle near the tip of the prosthesis, tensile stresses were reduced by $45 \%$, and shear stresses were reduced by $40 \%$. This stress reduction can increase the life of the implant by postponing fatigue failure. With the original $2 \mathrm{~mm}$ cement diameter, the modeled stresses exceeded the limit values for the prostheses. These stresses would cause premature fatigue failure. The study concluded that surgeons should use implants that allow a $5 \mathrm{~mm}$ cement mantle near the tip of the prosthesis. The smaller implant diameter will cause a more distributed load along the length of the prosthesis, rather than the majority of the load in the tip.

An article from 1998 discussed the different procedural factors

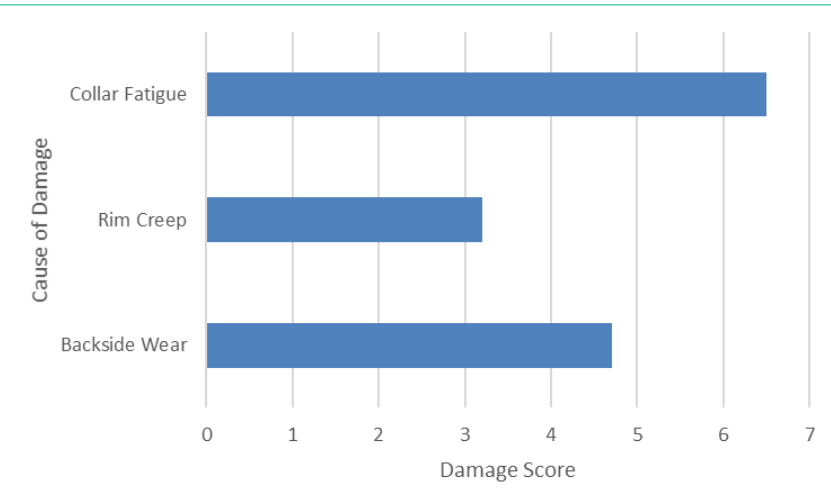

Figure 6: Damage of inlays due to different causes [9].

that affected the success of a cemented hip implant. It emphasized important factors involving the surgeon, the implant orientation, and the technique with which the cement was implemented [7]. The article suggested that previous studies have reported that cementing the acetabular component of the implant did not increase its lifespan or function. However, the article stated that with the correct technique and pressurization, the risk of loosening decreased. With regards to the femoral component of the implant, proper pressurization and cement texture was crucial to the successful hip replacement surgery. The surface of the femoral component on which the cement was bonded had a significant effect on the outcome of the surgery. Studies showed that the implants cemented to a rough surface had a lower success rate than using a smooth surface.

\section{Cementless hip implant failure}

In titanium cementless hip implants, the surfaces of both components are blasted to create a smooth gliding surface. A study done in 2002 looked at how the alumina-blasting process adds to the metal particle release of hip implants [8]. Early failure was reported in 7 alumina-blasted cementless hip implants due to pain and loosening. Upon further evaluation of the tissue surrounding the prosthesis, metal and alumina wear particles were found. The alumina-blasting process on titanium implants was determined to cause wear on the metal prostheses. This led to contamination of the metal due to surface scratches. The addition of alumina contamination along with metal particle release caused a greater incidence of implant failure and infection in the surrounding tissue.

In Germany, researchers analyzed 50 polyethylene inlays of cementless implants to examine the high failure rate of this specific design [9]. Different types of failure, such as creep, fatigue, wear, and deformation were evaluated and scored based on the extent of the damage present (Figure 6). The most common cause of failure was due to wear, which was present in $90 \%$ of the cases. In many instances, the space between the inlay and the head was not large enough to permit smooth movement. Normally, creep occurs only in the first two years, however, in these cases, creep was observed throughout the third year. It was concluded that the polyethylene inlay needs to be supported at the back to reduce wear and creep.

\section{Metal hip implant failure}

Three types of bearings were studied to determine which one had the best outcome in total hip arthroplasty [10]. These included metal-on-polyethylene, ceramic-on-ceramic, and metal-on-metal. 
When comparing the ceramic-on-ceramic bearing with the standard choice of metal-on-polyethylene bearing, the ceramic-on-ceramic bearing experienced a greater incidence of loosening, component failure, and pain. However, the metal-on-polyethylene bearings had a greater chance of infection and femur fracture. The metalon-metal bearings had a higher frequency of loosening, osteolysis, infection, femoral fracture, component failure, and pain. The metalon-polyethylene bearings had a greater incidence of dislocation when compared with the metal-on-metal bearings. Although metal-onpolyethylene bearings are the most popular choice for use in total hip arthroplasty, they generate a large number of wear particles that can lead to loosening of the prosthesis and, ultimately, failure. Ceramicon-ceramic bearings may be a more prevalent choice for younger patients because the greater amount of movement will not produce as much wear in the ceramic material. Metal-on-metal replacements are generally not used due to the multitude of failures that are more probable with metal. It is important for the surgeon to look at all of the options and discuss them with the patient to determine the best course of treatment for each specific case.

To help better understand the reason behind the failure of metalon-metal hip implants, a study was completed to assess the correlation between wear and corrosion, also called tribocorrosion, of a CoCrMo alloy [11]. Two different systems were utilized to complete this study. System A involved the linear movement of an aluminum ball against a flat metal surface. System B used an aluminum ball that rotated and was pressed against a cylindrical metal pin, which more closely emulates the hip joint movement. Both systems included a lubricant between the two metal components. The total mass lost in the metal was found and broken down into the amount that was due to wear and the amount that was due to corrosion. It was determined that System B had a lower amount of friction and a smaller mass loss per unit. This shows that during research, it is crucial to closely model the in vivo conditions to get the most accurate results.

\section{Other Causes of Hip Implant Failure}

\section{Effect of shape on implant failure}

A case study completed in 2013 followed a 47-year-old active patient who received a double-modular hip implant in 2006 [12]. After 3 years, the neck of the implant failed, and it was studied to determine why and how this type of implant can be improved. The removed prosthesis showed evidence of corrosion on the inside surfaces of the joint. There were also fatigue cracks visible on $94 \%$ of the fracture surface area. In double-modular implants, there was greatly reduced fatigue strength due to the corrosion caused by fluid penetration.

Between the years 2003 and 2011, patients who received hip implants were studied to determine the effectiveness of metal-onmetal bearings compared with ceramic-on-ceramic and metal-onpolyethylene [13]. To create a more uniform study, the patients included were suffering from osteoarthritis. An analysis was used to adjust patient age, and men were separated from women. Only about $8 \%$ of all hip replacements used a metal-on-metal bearing. It was also found that this type failed at a higher rate than the other two material implants. Another conclusion was that prosthesis with a larger head size was more likely to fail and require revision surgery for both men and women. However, when looking at ceramic-on-ceramic implants, there was a greater success using implants with larger head size.

\section{Acetabular component failure}

Failure of the acetabular component was studied to determine what type of revision surgery is the best option for implant survival [14]. A total of 664 revision surgeries were completed due to acetabular instability. The revision surgeries were divided into 3 groups: cemented polyethylene cups, metal rings, and non-cemented metal cups. It was determined that the best results were obtained with cemented and non-cemented implants where there were few acetabular defects. These groups provided a $91 \%$ success rate. When revision surgery is required, it is crucial to identify the problem as early as possible to minimize the amount of bone loss. The greater amount of acetabular bone defects, the greater chance of implant failure.

A study was completed in 1998 to determine the optimal angle of the acetabular component in a total hip arthroplasty [15]. The review included 75 patients with cemented hip replacements. Each implant in the study involved the same design and manufacturer to ensure comparable results. In the first group of patients, the acetabular component was inserted in such a way that the abduction angle was an average of 61.9 degrees. The second group of patients had an abduction angle averaging 49.7 degrees. Both groups had follow-ups within the same time frame so as not to alter the results. During the follow-up, the implant was checked for migration, loosening, and osteolysis. In all categories, the first group with the larger inclination angle had worse results than, the smaller inclination angle. A more horizontal angle of the acetabular component resulted in a greater success rate of hip prostheses.

\section{Femoral stem failure}

A case study of two failed metal hip implants that obtained a crack in the femoral neck was completed to determine the failure pathway [16]. The first patient encountered a fractured femoral neck 70 months after hip replacement. The second patient was found with a failed femoral neck 85 months after the operation. Both prostheses were examined using a scanning electron microscope and were found to have a corrosion-fatigue fracture. The failure of the femoral necks was caused by corrosion of the metal combined with the fatigue of the implants. Another factor that contributed to the failure was the manufacturing causing porous sections of the metal.

A study done on three different cases of stem failure in hip arthroplasty discussed the use of the Morse taper lock [17]. This mechanism involves a cone inserted into a sleeve. Research has been used to study the micromotion and corrosion of the Morse taper lock. These two scenarios have caused metal particles to be released into the surrounding body tissue, which can lead to infection or implant failure. Along with the Morse taper lock, modular femoral stems are becoming more commonplace and have been studied for failure. This 2009 study also looked at previous trials done to test the lifespan of modular hip implant components. It was concluded that failures occurred during torsional testing when the load was 6 times the body weight. Another study led to no failures due to torsion and there was no increase in osteolysis due to the modular implant component.

\section{Muscle atrophy with hip implants}

A study reviewed 56 patients to look for muscle atrophy, and if 


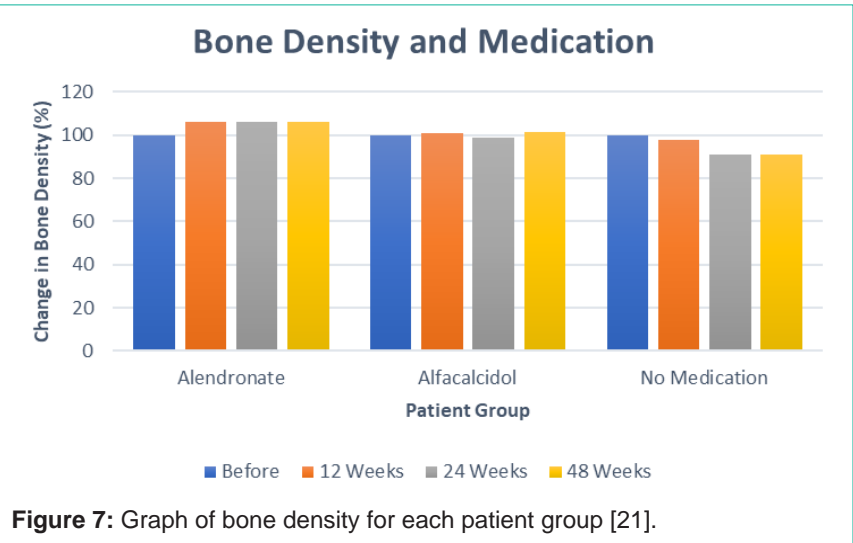

it progresses over time [18]. All patients received metal-on-metal implants and were studied using MRI around 83 months after surgery. Additional MRI scans were taken at an interval of 11 months. In all cases, the atrophy scores increased in both the gluteus minimus and gluteus medius. There was a significant increase in muscle atrophy from the first to the second MRI in the gluteus minimus and the posterior and inferior portions of the gluteus medius. Increased atrophy in the other muscles was not significant.

\section{Infection due to implant failure}

A common side effect of hip replacement surgery is a bacterial infection. The bacterium creates a film on the implant which then becomes resistant to antibiotics. A study was completed to determine whether adding gentamicin to the bone cement would prevent a bacterial infection [19]. There were 20 specimens collected from patients that received a revision surgery. Gentamicin was added to the bone cement and then used on the gathered specimen. After 72 hours, there was no difference in film formation between the gentamicin and non-gentamicin cemented specimens. However, when an antibiotic was administered before and after surgery, there was less likelihood of infection occurring. It was concluded that the combination of gentamicin and the added antibiotic were the best defense against bacteria forming on the implant.

Some patients cannot undergo an implant removal surgery to treat an infected hip prosthesis. An alternative solution is to administer antibiotics, debride the infection, and keep the implant. The efficacy of this procedure is dependent upon the type of bacteria causing the infection. A study was completed to determine whether this treatment worked best for Staphylococcus aureus or Streptococcus [20]. This study looked at hip implants infected with Staphylococcus aureus and Streptococcus that were treated with antibiotic and debridement treatment. The patients were followed up with around 3.5 years after treatment, and it was found that the streptococcal infections had a lower failure rate. With treatment, the Staphylococcus aureus infections had a failure rate of $21 \%$, which was much higher than Streptococcus.

\section{Medication use and implant failure}

A study, which was completed in 2011, compared the effect of the bisphosphonate derivative, alendronate with the vitamin $\mathrm{D}$, alfacalcidol [21]. Both of these medications were used to improve bone density and the research aimed to show which one improved

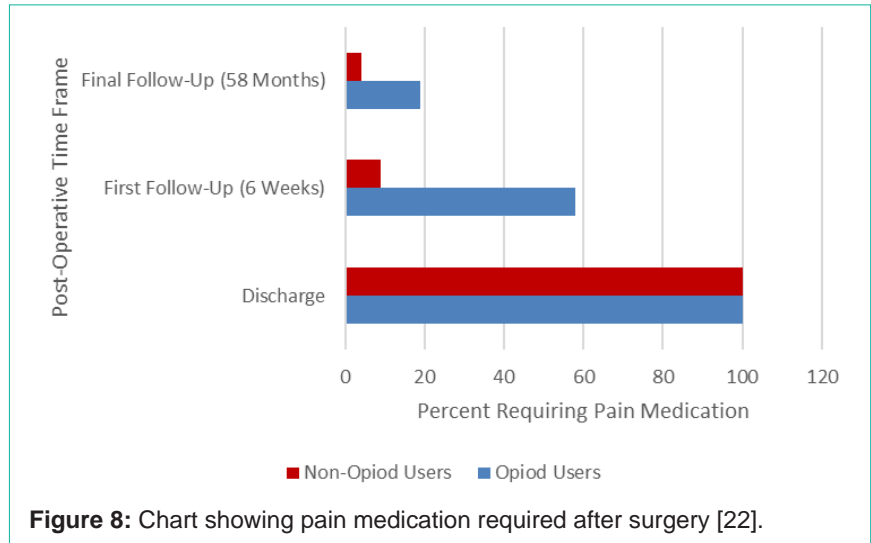

the lifespan of the hip implant following a total hip arthroplasty. Patients began taking the selected medication the first day after the operation and continued for 48 weeks. There were 20 patients taking alendronate, 18 patients took alfacalcidol, and 22 patients did not take any medication. Bone density measurements were taken for all patients one week after surgery as a baseline and then at 12,24 , and 48 weeks after the operation. The results demonstrate that the patient group with no medication experienced decreasing bone density with time (Figure 7). With alfacalcidol, the patients had a slight loss in bone density at 24 weeks postoperatively, but it increased again at 48 weeks. The most successful treatment was achieved with alendronate. This medication increased bone density and that value were maintained throughout the study.

Similar research was done to compare the hip implant surgery outcome between patients using opioid medication 3 months before surgery and patients not using opioids for pain [22]. In each group, there were a total of 54 patients and each group was followed-up 6 weeks and then 5 years after surgery. It was determined that the patients using opioids for osteoarthritis prior to surgery experienced a longer hospital stay and required more pain medications after surgery (Figure 8). Along with these two results, the patients on opioids also had worse clinical outcomes demonstrated by a lower Harris hip score. The Harris hip score measures hip function and patient pain level after total hip arthroplasty.

\section{Obesity and implant failure}

A research study published in 2005 focused on the effect of a $\mathrm{BMI}>30$ and the early outcome of hip replacement [23]. Obesity is considered when determining postoperative complications such as deep venous thrombosis and wound infection. In this study, a group of obese patients was directly compared to a group of patients with a normal BMI. The obese group consisted of 151 patients, while the normal BMI group consisted of 162 patients. One year after the total hip arthroplasty, patients filled out a questionnaire. Both groups had the patient satisfaction of greater than $90 \%$. Each group underwent 5 revision surgeries, which demonstrated no significant difference in the obese patients and normal BMI patients (Figure 9).

\section{Implant failure and diabetes}

An investigation focused on the correlation between diabetes and hip implant failure [24]. A total number of 69 patients with diabetes were included in the research study. Of these, 17 patients were 


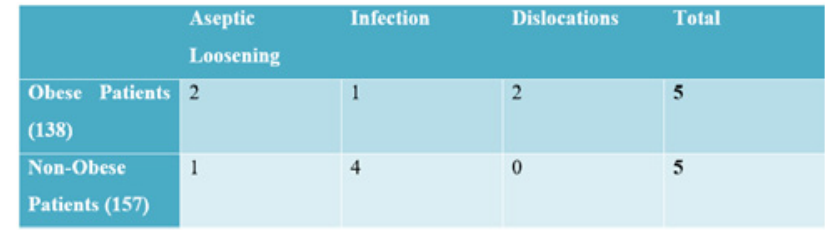

Figure 9: Number of revisions required for both groups.

insulin-dependent, while 52 patients were noninsulin-dependent. After an average follow-up time of 4 years, there was a $3.7 \%$ revision rate. Radiographs showed loosening in $22.2 \%$ of the acetabular cups. Regarding infection, there was a $14.2 \%$ incidence of urinary tract infection for all of the patients. This proved to be the most prevalent cause of morbidity in this study.

Another study focused on the risk of aseptic loosening in patients with diabetes [25]. A total of 16,085 total joint arthroplasties were completed for this study, and 2,911 patients had diabetes. This study included both hip and knee arthroplasties. After a follow-up of around 6 years, 25 patients with diabetes underwent revision surgery due to aseptic loosening. In patients without diabetes, 157 surgeries were needed for aseptic loosening. These results show that there is no significant difference in aseptic loosening between patients with and without diabetes.

\section{Implant failure and the lymphatic system}

When hip implants loosen inside the body, material from the prosthetic enters the surrounding tissues. A study conducted on hip implant revisions focused on how the lymphatic system contributes to inflammation caused by implant failure [26]. Tissue samples near the bone-implant interface were taken and tested for podoplanin, a blood protein that indicates lymphatic vessel formation. It was found that there were a low number of podoplanin-positive vessels near the bone-implant interface. The consequences of inadequate vessels were determined to be inflammation and edema. These conditions would be caused by insufficient vessels to remove interstitial fluids and cells.

Research was completed to determine the effect of hip implant particles on the abdominal lymph nodes, liver, and spleen [27]. Specimens were obtained from 29 cadavers and 2 living patients who had failed implants. In $89 \%$ of the patients, metallic or polyethylene particles were identified in the para-aortic abdominal lymph nodes. Also, $38 \%$ of patients had metallic particles travel to the liver or spleen. Large amounts of particles in the lymph nodes led to fibrosis, histiocytosis, and lymph node necrosis. These issues caused immune activation and the production of cytokines.

\section{Implant failure associated with smoking}

A study was completed to determine the association between smoking and hip implant failure. The revision rates of metal-onmetal implants were compared to the revision rates of ceramic-onpolyethylene implants [28]. A total of 663 patients had metal-onmetal bearings, while 1,301 patients had ceramic-on-polyethylene bearings. In the metal-on-metal group, there were 18 required revisions among smokers, and only 4 required revisions among nonsmokers. The patients were evaluated after about 7 years. For the ceramic-on-polyethylene group, there were 16 revisions for smokers and 18 revisions for nonsmokers. This data showed that smoking was linked to a higher failure rate of patients who had received a metalon-metal hip implant. However, there was no correlation between smoking and ceramic-on-polyethylene implant failure.

An investigation of early failure after hip implants was done to determine a correlation between smoking and short-term failure [29]. There were a total of 78,191 patients included in the study. Of this group, 8,062 were current smokers, 6,158 were former smokers, and 63,971 were nonsmokers. The patient progress was studied 30 days postoperatively. Analysis after operation showed that current smokers were twice as likely as former and nonsmokers to experience deep wound infection. These results affirm the previous findings that smoking leads to poor wound healing. Another result led to the conclusion that former smokers had an increased risk of short-term morbidity and mortality when compared to current and nonsmokers.

\section{Effect of gender on implant failure}

A study conducted in 2013 looked at how the gender of a patient affects the success rate of a total hip arthroplasty [30]. Out of 35,140 implants, $57.5 \%$ were women, and $42.5 \%$ were men. The women more often had a metal-on-polyethylene type and smaller femoral head size of $28 \mathrm{~mm}$. The men had a metal-on-metal implant with a larger femoral head size of $36 \mathrm{~mm}$. The experience levels of the surgeons were comparable in all cases to minimize outside factors affecting the outcomes. Along with this, the main diagnosis was osteoarthritis, and the average patient age was between 60 and 69 years. After a followup of approximately 3 years, women had a higher risk of requiring revision surgery due to loosening, infection, and instability among others. This study would not be as reliable due to the fact that the men received a different material implant than the women. This caused the results not to be directly comparable.

A more specific study focused on the success differences between men and women who received metal-on-metal hip implants [31]. This material has been a popular choice due to low wear and increased stability. From a total of 1,367 patients studied, 719 were male, and 643 were female. After an average follow-up time of 60 months, there were 65 failures. Of these, 49 were due to loosening or metal complications. Also, 48 of the failures occurred in women. There was no difference in the failures vs. survivors for women regarding implant size or inclination angle. This concludes that there are additional factors for women that lead to greater failure rate. These could include anatomic, hormone, or functional differences.

\section{Conclusion}

The results of the studies indicate that the metal-on-polyethylene and the metal-on-ceramic implants have brought about the greatest successes. These two types have become the most popular implant designs in recent years due to the discovery of metal particle release and other side effects. Cemented implants are more successful with smooth surface prostheses because the rough surface may wear the cement if it becomes de bonded. When considering the shape of hip implants, a more horizontal angle of the stem leads to a more positive result and greater range of motion. In the end, it is crucial for the surgeon to research the different types of implants and discuss with the patient what the best choice may be to ensure the most effective outcome.

With regards to patient risk factors, smoking and medication 
use are often associated with a greater failure rate after total hip arthroplasty. Certain medications are linked to decreased bone density and should be considered prior to hip surgery. Surprisingly, obesity does not have a significant impact on implant failure. Also, some patients experienced muscle atrophy of the gluteus minimus or gluteus medius. However, there were no factors that would suggest more susceptibility to muscle atrophy. Each patient needs to be evaluated individually to determine specific risk factors and to be monitored closely after surgery. Throughout the past 30 years, there has been much research in total hip arthroplasties and the best options for success. The studies have suggested that each type of implant has benefits, but it is important to study the failures as well. With a better understanding of failure, safer and more durable implants can be developed in the future.

\section{References}

1. Knight SR, Aujla R, Biswas SP. Total Hip Arthroplasty - over 100 years of operative history. Orthop Rev. 2011; 3: e16.

2. Pazzaglial UE, Ceciliani L, Wilkinson MJ, Dell'Orboz C. Involvement of metal particles in loosening of metal-plastic total hip prostheses. Arch Orthop Trauma Surg. 1985; 104: 164-174.

3. Paustenbach DJ, Galbraith DA, Finley BL. Interpreting cobalt blood concentrations in hip implant patients. Clin Toxicol. 2014; 52: 98-112.

4. Ovid: Osteolysis in Association with a Total Hip Arthroplasty with Ceramic Bearing Surfaces*. (Accessed: 24th June 2017)

5. Brandt JM, Gascoyne TC, Guenther LE, Allen A, Hedden DR, Turgeon TR, et al. Clinical failure analysis of contemporary ceramic-on-ceramic total hip replacements. Proc Inst Mech Eng [H]. 2013; 227: 833-846.

6. Lee IY, Skinner HB, Keyak JH. Effects of variation of prosthesis size on cement stress at the tip of a femoral implant. J Biomed Mater Res. 1994; 28 1055-1060.

7. Crawford RW, Murray D. (iv) Early failure of cemented designs. Curr Orthop. 1998; 12: 244-250.

8. Böhler M, Kanz F, Schwarz B, Steffan I, Walter A, Plenk H, et al. Adverse tissue reactions to wear particles from Co-alloy articulations, increased by alumina-blasting particle contamination from cementless Ti-based total hip implants. A report of seven revisions with early failure. J Bone Joint Surg Br. 2002; 84: 128-136.

9. Mueller U, Lee C, Heisel C, Thomsen M, Bitsch RG, Kretzer JP. Failure of Polyethylene Inlays in Cementless Total Hip Arthroplasty: A Retrieval Analysis. BioMed Res Int. 2016

10. Varnum C. Outcomes of different bearings in total hip arthroplasty - implant survival, revision causes, and patient-reported outcome. Dan Med J. 2017

11. Mathew MT, Runa MJ, Laurent M, Jacobs JJ, Rocha LA, Wimmer MA Tribocorrosion behavior of CoCrMo alloy for hip prosthesis as a function of loads: A comparison between two testing systems. Wear. 2011; 271: 12101219.

12. Williams JJ, Chawla N. Fractography of a neck failure in a double-modular hip implant. Case Stud Eng Fail Anal. 2014; 2: 45-50.

13. Smith AJ, Dieppe P, Vernon K, Porter M, Blom AW. Failure rates of stemmed metal-on-metal hip replacements: analysis of data from the National Joint Registry of England and Wales. The Lancet. 2012; 379: 1199-1204.

14. Zagorodniy N, Nikolaev I, Nuzhdin V, Kagramanov S. Prospective cohort study of six hundred and sixty four revisions of loose failed acetabular implants. Int Orthop. 2014; 38: 2021-2025

15. Kennedy JG, Rogers WB, Soffe KE, Sullivan RJ, Griffen DG, Sheehan LJ. Effect of acetabular component orientation on recurrent dislocation, pelvic osteolysis, polyethylene wear, and component migration. J Arthroplasty. 1998; 13: 530-534.

16. Gilbert JL, Buckley CA, Jacobs JJ, Bertin KC, Zernich MR. Intergranula corrosion-fatigue failure of cobalt-alloy femoral stems. A failure analysis of two implants. J Bone Joint Surg Am. 1994; 76: 110-115.

17. Patel A, Bliss J, Calfee RP, Froehlich J, Limbird R. Modular Femoral StemSleeve Junction Failure After Primary Total Hip Arthroplasty. J Arthroplasty. 2009; 24: 1143.e1-1143.e5.

18. Berber R, Khoo M, Cook E, Guppy A, Hua J, Miles J, et al. Muscle atrophy and metal-on-metal hip implants. Acta Orthop. 2015; 86: 351-357.

19. Tunney MM, Dunne N, Einarsson G, McDowell A, Kerr A, Patrick S. Biofilm formation by bacteria isolated from retrieved failed prosthetic hip implants in an in vitro model of hip arthroplasty antibiotic prophylaxis. J Orthop Res. 2007; 25: 2-10.

20. Betz M, Abrassart S, Vaudaux P, Gjika E, Schindler M, Billières J, et al. Increased risk of joint failure in hip prostheses infected with Staphylococcus aureus treated with debridement, antibiotics and implant retention compared to Streptococcus. Int Orthop. 2015; 39: 397-401.

21. Iwamoto $N$, naba $Y$, Kobayashi $N$, Ishida $T$, Yukizawa $Y$, Saito $T$. A Comparison of the Effects of Alendronate and Alfacalcidol on Bone Mineral Density Around the Femoral Implant and in the Lumbar Spine After Total Hip Arthroplasty: J Bone Jt Surg Am. 2011; 93: 1203-1209.

22. Pivec, R, Issa K, Naziri Q, Kapadia BH, Bonutti PM, Mont MA. Opioid use prior to total hip arthroplasty leads to worse clinical outcomes. Int Orthop. 2014; 38: 1159-1165.

23. Ibrahim T, Hobson S, Beiri A, Esler CN. No influence of body mass index on early outcome following total hip arthroplasty. Int Orthop. 2005; 29: 359-361.

24. Moeckel B, Huo MH, Salvati EA, Pellicci PM. Total hip arthroplasty in patients with diabetes mellitus. J Arthroplasty. 1993; 8: 279-284

25. Maradit Kremers $H$, Schleck CD, Lewallen EA, Larson DR, Van Wijnen AJ, Lewallen DG. Diabetes Mellitus and Hyperglycemia and the Risk of Aseptic Loosening in Total Joint Arthroplasty. J Arthroplasty. 2017.

26. Jell G, Kerjaschki D, Revell P, Al-Saffar N. Lymphangiogenesis in the boneimplant interface of orthopedic implants: Importance and consequence. J Biomed Mater Res A. 2006; 77: 119-127.

27. Ovid: Dissemination of Wear Particles to the Liver, Spleen, and Abdominal Lymph Nodes of Patients with Hip or Knee Replacement*. (Accessed: 17th July 2017)

28. Lübbeke A, Rothman KJ, Garavaglia G, Barea C, Christofilopoulos P, Stern $\mathrm{R}$, et al. Strong association between smoking and the risk of revision in a cohort study of patients with metal-on-metal total hip arthroplasty. J Orthop Res. 2014; 32: 762-768.

29. Duchman KR, Gao Y, Pugely AJ, Martin CT, Noiseux NO, Callaghan JJ. The Effect of Smoking on Short-Term Complications Following Total Hip and Knee Arthroplasty. J Bone Jt Surg. 2015; 97: 1049-1058.

30. Inacio MC, Ake CF, Paxton EW, Khatod M, Wang C, Gross TP, et al. Sex and Risk of Hip Implant Failure: Assessing Total Hip Arthroplasty Outcomes in the United States. JAMA Intern. Med. 2013; 173: 435-441.

31. Latteier MJ, Berend KR, Lombardi AV, Ajluni AF, Seng BE, Adams JB. Gende is a Significant Factor for Failure of Metal-on-Metal Total Hip Arthroplasty. J Arthroplasty. 2011; 26: 19-23.
Austin J Orthopade \& Rheumatol - Volume 5 Issue 1 - 2018 ISSN: 2472-369X | www.austinpublishing group.com Mathew et al. (C) All rights are reserved
Citation: Sipek KT, Lyvers ME and Mathew MT. Failure Causes in Total Hip Replacements: A Review. Austin J Orthopade \& Rheumatol. 2018; 5(1): 1064. 